\title{
Duration of Methicillin-Resistant Staphylococcus aureus Carriage, According to Risk Factors for Acquisition
}

\author{
Jonas Marschall, MD; Kathrin Mühlemann, MD, PhD
}

ов Jест IVE. To examine the duration of methicillin-resistant Staphylococcus aureus (MRSA) carriage and its determinants and the influence of eradication regimens.

DESIGN. Retrospective cohort study.

SETtING. A 1,033-bed tertiary care university hospital in Bern, Switzerland, in which the prevalence of methicillin resistance among $S$. aureus isolates is less than $5 \%$.

PATIENTs. A total of 116 patients with first-time MRSA detection identified at University Hospital Bern between January 1,2000 , and December 31, 2003, were followed up for a mean duration of 16.2 months.

RESULTS. Sixty-eight patients (58.6\%) cleared colonization, with a median time to clearance of 7.4 months. Independent determinants for shorter carriage duration were the absence of any modifiable risk factor (receipt of antibiotics, use of an indwelling device, or presence of a skin lesion) (hazard ratio [HR], 0.20 [95\% confidence interval $\{\mathrm{CI}\}, 0.09-0.42]$ ), absence of immunosuppressive therapy (HR, 0.49 [95\% CI, 0.23-1.02]), and hemodialysis (HR, 0.08 [95\% CI, 0.01-0.66]) at the time MRSA was first MRSA detected and the administration of decolonization regimen in the absence of a modifiable risk factor (HR, 2.22 [95\% CI, 1.36-3.64]). Failure of decolonization treatment was associated with the presence of risk factors at the time of treatment $(P=.01)$. Intermittent screenings that were negative for MRSA were frequent ( $26 \%$ of patients), occurred early after first detection of MRSA (median, 31.5 days), and were associated with a lower probability of clearing colonization ( $\mathrm{HR}, 0.34$ [95\% CI, 0.17-0.67]) and an increased risk of MRSA infection during follow-up.

CONCLUSIONs. Risk factors for MRSA acquisition should be carefully assessed in all MRSA carriers and should be included in infection control policies, such as the timing of decolonization treatment, the definition of MRSA clearance, and the decision of when to suspend isolation measures.

Infect Control Hosp Epidemiol 2006; 27:1206-1212

Methicillin-resistant Staphylococcus aureus (MRSA) is a major cause of nosocomial infections worldwide, resulting in substantial morbidity and mortality. Humans are the only significant reservoir of MRSA. Colonization with the microorganism is a necessary step during the pathogenesis of MRSA infection, and it is the source of cross-transmission between humans. ${ }^{1,2}$ Placement of MRSA carriers in contact isolation during their stay in healthcare institutions is a current standard of care. ${ }^{3,4}$ Although cost-effective overall, isolation measures are expensive. A recent retrospective study calculated an additional cost of $\$ 318$ per hospitalization-day per patient with MRSA, of which approximately $80 \%$ was from the use of barrier precautions around beds in multiple-bed rooms. ${ }^{5}$ Breaks in compliance with isolation policies are a continuous threat for the dissemination of MRSA.

Colonization may persist for months or even years. ${ }^{6-8} \mathrm{How}-$ ever, data are insufficient about the natural history of MRSA colonization and its determinants. Furthermore, the role of topical decolonization therapy is still controversial. ${ }^{9-11}$ A large meta-analysis concluded that there is no evidence that treatment with antimicrobials can eradicate MRSA carriage. ${ }^{12}$

In the present study, we sought to determine the duration of MRSA carriage and how the duration is influenced by risk factors for MRSA acquisition and receipt of decolonization treatment. The rationale was to create a basis for the optimal timing for initiating decolonization treatment and suspending isolation measures for MRSA carriers.

\section{METHODS}

\section{Study Setting and Local Infection Control Policy}

University Hospital Bern (Bern, Switzerland) is a 1,033-bed tertiary care center with more than 30,000 admissions per year. The referral area covers the local region, where approximately 1 million inhabitants live, and a large number of smaller hospitals within and outside the Bernese region. In this part of Switzerland, the prevalence of methicillin re-

From the Department of Infectious Diseases, University Hospital Bern (J.M., K.M.), and Institute for Infectious Diseases, University of Bern (K.M.), Bern, Switzerland.

Received April 12, 2006; accepted June 14, 2006; electronically published October 4, 2006.

(C) 2006 by The Society for Healthcare Epidemiology of America. All rights reserved. 0899-823X/2006/2711-0011\$15.00. 
sistance among S. aureus isolates is less than $5 \%,{ }^{13}$ and it ranges from $3 \%$ to $5 \%$ at University Hospital Bern.

According to local written infection control guidelines, MRSA carriers are put into contact isolation, which involves stay in a single-patient room, use of gloves and gowns by medical personnel during physical contact, and use of masks by medical personnel when exposure to respiratory secretions is expected. In the interdisciplinary intensive care unit, contact isolation is implemented in 4-bed cubicles by marking an isolation area of approximately $2 \mathrm{~m}$ around the patient's bed with paravents and closing the neighboring bed. Screening for MRSA involves all patients in contact with an MRSA carrier for whom placement in isolation was delayed and patients treated in a foreign healthcare institution during the 6 months before admission. Patients with MRSA are tagged in the hospital's patient administration system. On readmission, tagged MRSA carriers are put into contact isolation.

MRSA decolonization treatment comprises a 5-day course of nasal application of mupirocin ointment, daily skin disinfection with $4 \%$ chlorhexidine soap, and daily gargling with $0.1 \%$ chlorhexidine solution. The decision for decolonization treatment is made by the infection control team and requires that systemic antibiotic therapy and indwelling devices have not been used for at least 14 days and that all skin lesions have healed. During the study period, screening for MRSA control was performed intermittently as convenient during ambulatory visits or inpatient treatment, and results were therefore distributed over intervals of weeks to months, independent of whether decolonization treatment had been administered.

\section{Recruitment of Study Patients and Collection of Data}

The study was performed in accordance with local ethical guidelines. Patients with MRSA carriage detected for the first time between January 1, 2000, and December 31, 2003, were identified retrospectively through the laboratory information system of the Institute for Infectious Diseases, University of Bern, which serves University Hospital Bern. A total of 223 patients met these criteria. Thirty-eight patients were excluded, because they were not treated at University Hospital Bern at the time MRSA was first detected, and information concerning risk factors for MRSA acquisition was not available. An additional 69 patients were lost to follow-up after 1 month and were therefore also excluded. A total of 116 patients remained for analysis.

A standardized questionnaire was used to collect patient data retrospectively from medical records, infection control unit statistics, and the laboratory information system. Followup for individual patients started on the date MRSA was first detected and ended on December 31, 2004.

\section{Terms and Definition}

The following risk factors for MRSA carriage at the time MRSA was first detected were considered: receipt of systemic antibiotic therapy or performance of a surgical procedure during the last 30 days, presence of skin lesions (surgical site wounds, ulcers, and/or dermatitis), use of indwelling devices (intravascular central catheters, endotracheal tube or tracheostoma, urinary catheters, and/or wound drainage devices), receipt of immunosuppressive therapy (steroid treatment equivalent to $7.5 \mathrm{mg}$ /day or more of prednisone, chemotherapy, or immunomodulators), presence of diabetes mellitus, presence of renal insufficiency, receipt of hemodialysis, and presence of malignant tumors. Risk factors considered during follow-up were the same as those for the time when MRSA was first detected, with the exception of performance of surgical interventions during the past 30 days. We calculated the sum of risk factors present at the following junctures: the time MRSA was first detected, the time of decolonization treatment (if applicable), and the time of the final MRSA screening during follow-up. For analysis, risk factors were also categorized dichotomously (3 or less vs more than 3 ). Moreover, we defined modifiable risk factors as receipt of antibiotic treatment within the previous 30 days, presence of skin lesions, and use of indwelling foreign devices.

The total follow-up time was defined as the interval from the first detection of MRSA until the last MRSA screening (positive or negative). Clearance of MRSA carriage was assumed when 2 or more of the final screenings during follow-up were negative for MRSA. Long-term carriage was defined as persistent carriage for more than 12 months during follow-up.

\section{Detection of and Screening for MRSA Colonization}

Routine MRSA screening involved testing of specimens from the following anatomical sites: both nares, the groin (2 specimens), any skin lesions, tracheal secretions in intubated or tracheostomized patients, and urine in patients with urinary catheter. Samples obtained for suspected MRSA infection were included in the screening samples. Swabbing was performed with twisted wire rayon-tipped applicators (Copan Venturi Transystem). One applicator was used for both nares and another for both specimens from the groin, and applicators were premoistened with physiological saline. Laboratory screening for MRSA was performed with the mannitoloxacillin biplate test, selective culturing for gram-positive organisms, the coagulase tube test (to exclude coagulasenegative staphylococcus), and resistance pattern testing on Müller-Hinton agar. Available consecutive MRSA isolates from the same patient were typed using pulsed-field gel electrophoresis (PFGE), as described elsewhere. ${ }^{14}$

\section{Statistical Analysis}

All analyses were performed in StatView, version 5.0 (SAS Institute), or Stata, version 8 (Stata Corporation), using a cutoff of $P$ value of .05 or less (2-tailed). Differences between means were tested by the Student's $t$ test or the Mann-Whitney $U$ test, and proportions were compared with the $\chi^{2}$ or Fisher's exact test, as appropriate. The duration of MRSA 
colonization determinants was analyzed by Kaplan-Meier curves using the log-rank test and Cox regression analysis.

\section{RESULTS}

\section{Characteristics of Study Patients and Risk Factors for MRSA Acquisition}

A total of 116 patients with newly detected MRSA colonization were followed up for a mean duration $( \pm S D)$ of $496 \pm 431$ days (median, 316.5 days [range, 31-1,616 days]). The characteristics of these 116 patients at the time MRSA was first detected are listed in Table 1. Almost half of the patients $(45.7 \%)$ were 65 years or older. Male patients predominated $(69.8 \%$ of the study population). Most patients were of Swiss nationality $(80.2 \%)$ and lived in private households (90.5\%). Half of the MRSA carriers (48.3\%) were identified through the screening of contacts. The predominant anatomical sites of MRSA detection were skin lesions (39.7\%) and the nares $(35.3 \%)$. The most prevalent risk factors for MRSA acquisition were skin lesions (71.6\% of patients), past antibiotic therapy (56.9\%), and past surgery (54.3\%). Almost all patients $(96.6 \%)$ had been hospitalized within the previous 6 months, and the mean hospitalization duration $( \pm S D)$ before MRSA detection was $11.9 \pm 17.7$ days. Almost half of the patients $(43.1 \%)$ had an MRSA infection at the time MRSA was first detected.

\section{Clearance of MRSA During Follow-up}

During follow-up, a mean of 3.8 MRSA screenings were performed per patient per year. Sixty-eight patients (58.6\%) cleared MRSA colonization. The median time to clearance, based on the Kaplan-Meier estimate, was 226 days (7.4 months). In patients who cleared colonization, the median follow-up time after clearance was 189.5 days (range, 1-1,602 days). Long-term carriage was observed in 24 patients (20.7\%), of whom 19 cleared MRSA colonization, and 5 remained colonized throughout the follow-up period.

Independent determinants for MRSA clearance were the absence of modifiable risk factors (hazard ratio [HR], 0.20 [95\% confidence interval $\{\mathrm{CI}\}, 0.09-0.42$ ]; $P<.001$ ), receipt of immunosuppressive therapy (HR, 0.49 [95\% CI, $0.23-$ 1.02 ]; $P=.05$ ), and hemodialysis (HR, 0.08 [95\% CI, 0.010.66 ]; $P=.01$ ) at the time MRSA was first detected and the administration of decolonization treatment (HR, $2.22[95 \%$ $\mathrm{CI}, 1.36-3.64] ; P=.01$ ) (Table 2). Thirty-six patients $(31.0 \%)$ received decolonization treatment, which failed in $13(36.1 \%)$. The median time to initiation of decolonization treatment was 37.5 days (range, 0-833 days). The median time to clearance (based on the Kaplan-Meier estimate) for patients with $0-3$ risk factors at the time of MRSA detection was 43 days if decolonization treatment was administered and 238 days if it was not administered (Figure). The corresponding intervals for patients with more than 3 risk factors were 221 days if decolonization treatment was received and 597 days if it was not received. The 13 patients who did not respond to decolonization treatment had a significantly greater number of risk factors at the time of decolonization treatment (mean $[ \pm \mathrm{SD}], 1.30 \pm 1.37$ [95\% CI, 0.47-2.14]) than patients who cleared MRSA immediately after treatment $(0.39$ \pm 0.65 risk factors [95\% Cl, 0.11-0.67]; $P=.01$ ).

\section{Intermittent Negative Results of Screenings for MRSA}

During follow-up, 30 patients $(25.9 \%)$ had intermittent screenings with negative results, and 12 patients $(10.3 \%)$ had 2 or more consecutive intermittent screenings with negative results. Twelve of the 30 patients had PFGE performed on MRSA isolates that were obtained before and after intermittent screenings with negative results; for all 12, the PFGE pattern for the first isolate matched the pattern for the second isolate. For 3 of the 12 patients, extended intervals of 33, 39, and 51 months occurred between the times the 2 MRSA isolates with an identical PFGE pattern were obtained. The median interval between the time MRSA was first detected to the time of the first intermittent screening with a negative result in these 30 patients was 31.5 days (range, 1-365 days), compared with the median time to MRȘA clearance of 226 days in the whole study population. The mean number of risk factors present at the time MRSA was first detected was higher for patients with negative results of intermittent screenings (mean, 3.86 vs $2.96 ; P=.002$ ), and they had a lower probability of clearing MRSA colonization (Table 2). In the Cox regression analysis, the only independent factor associated with negative results of intermittent screenings was the number of risk factors present at time MRSA was first detected (HR, 1.65; 95\% CI, 1.24-2.20; $P=.005$ ).

\section{MRSA Infection During Follow-up}

Of 116 patients, 17 (14.7\%) developed an MRSA infection during follow-up. Five of the 17 patients also had an MRSA infection at the time MRSA was first detected. Infection occurred a median interval of 123 days (range, 4-705 days; mean interval [ $\pm S D$ ], $192.5 \pm 212.3$ days) after MRSA was first detected. Kaplan-Meier estimates could not be obtained, because less than $50 \%$ of patients developed MRSA infection. In the Cox regression analysis, only a large number of risk factors present at the time of the last MRSA screening (HR, 1.41 [95\% CI, 1.009-1.975]; $P=.04$ ) was significantly associated with MRSA infection. None of the other variables reached statistical significance, perhaps in part because of the small number of MRSA infections observed.

\section{DISCUSSION}

The time course of MRSA colonization and its determinants was described in a retrospective cohort of 116 patients with MRSA at a tertiary care university hospital. The characteristics of the study population corresponded to known risk factors for MRSA acquisition, such as older age, prior hospitalization (especially in the intensive care unit), previous surgery, previous antibiotic treatment, presence of skin lesions, and use 
TA B LE 1. Characteristics of 116 Patients Colonized With MethicillinResistant Staphylococcus aureus (MRSA) at University Hospital Bern (Bern, Switzerland) between January 1, 2000, and December 31, 2003

\begin{tabular}{l} 
Characteristic \\
\hline Age \\
Mean y $\pm S D$ \\
$0-16 y$ \\
$17-44 y$ \\
$45-64 y$ \\
$\geqslant 65 y$
\end{tabular}

Male sex

Residence

Private household

Long-term care facility

Immigrant housing facility

Method of MRSA detection

Clinical diagnostic workup

MRSA screening, by patient group ${ }^{a}$

Overall

Contacts with an MRSA carrier

Patients admitted from a foreign institution

Anatomical site of MRSA detection ${ }^{b}$

Skin lesion or wound

Nose

Urine

Groin

Respiratory tract

Risk factor for MRSA acquisition ${ }^{\mathrm{b}}$

Skin lesion(s)

Surgery during past $30 \mathrm{~d}$

Antibiotic therapy during past $12 \mathrm{mo}^{\mathrm{c}}$

During past $30 \mathrm{~d}$

During past 1-12 mo

Indwelling device

Immunosuppressive therapy

Diabetes mellitus

Renal insufficiency

Hemodialysis

Malignant disease

Hospitalization history

Stay of $\geqslant 24 \mathrm{~h}$ during past $6 \mathrm{mo}$

Length of stay, mean $d \pm S D$

MRSA detected during stay

Length of stay before MRSA detection, mean $\mathrm{d} \pm \mathrm{SD}$

$55.7 \pm 21.4$
$6(5.2)$
$26(22.4)$
$31(26.7)$
$53(45.7)$
$81(69.8)$

$105(90.5)$

$7(6.0)$

$4(3.4)$

$60(51.7)$

$56(48.3)$

$48(41.4)$

$8(6.9)$

$46(39.7)$

$41(35.3)$

$15(12.9)$

$12(10.3)$

$8(6.9)$

$83(71.6)$

$63(54.3)$

$77(66.4)$

$66(56.9)$

$30(25.8)$

$53(45.7)$

$23(19.8)$

$23(19.8)$

$21(18.1)$

$7(6.0)$

$23(19.8)$

112 (96.6)

$21.9 \pm 22.2$

$92(79.3)$

$11.9 \pm 17.7$

$46(39.7)$

Admitted during current hospitalization

Length of stay before MRSA detection, mean $\mathrm{d} \pm \mathrm{SD}$

MRSA infection at time of first MRSA detection

Absent

Subsequent infection during follow-up

Time to subsequent onset, mean $d \pm S D$
TABLE 1. (Continued)

\begin{tabular}{|c|c|}
\hline Characteristic & Value \\
\hline Present, by infection location & $50(43.1)$ \\
\hline Surgical site & $24(48.0)$ \\
\hline Skin or soft tissue & $8(16.0)$ \\
\hline Urinary tract & $6(12.0)$ \\
\hline Bone or prosthesis & $5(10.0)$ \\
\hline Respiratory tract & $3(6.0)$ \\
\hline Abdominal & $2(4.0)$ \\
\hline Sepsis & $1(2.0)$ \\
\hline Peridural catheter & $1(2.0)$ \\
\hline Surgery for MRSA infection & $29(58.0)$ \\
\hline \multicolumn{2}{|l|}{$\begin{array}{l}\text { MRSA infection during follow-up, } \\
\text { by infection location }\end{array}$} \\
\hline Overall & $17(14.7)$ \\
\hline Surgical site & $3(17.6)$ \\
\hline Skin or soft tissue & $5(29.4)$ \\
\hline Urinary tract & $3(17.6)$ \\
\hline Bone or prosthesis & $1(5.9)$ \\
\hline Respiratory tract & $1(5.9)$ \\
\hline Sepsis & $4(23.5)$ \\
\hline \multicolumn{2}{|l|}{ Duration of follow-up } \\
\hline Mean $d \pm S D$ & $496.0 \pm 431.4$ \\
\hline$<3 \mathrm{mo}$ & $20(17.2)$ \\
\hline $3-6 \mathrm{mo}$ & $20(17.2)$ \\
\hline$>6-24 \mathrm{mo}$ & $40(34.5)$ \\
\hline$>24-48 \mathrm{mo}$ & $34(29.3)$ \\
\hline$>48 \mathrm{mo}$ & $2(1.7)$ \\
\hline
\end{tabular}

NOTE. Data are no. (\%) of patients, unless otherwise indicated. ICU, intensive care unit; MRSA, methicillin-resistant Staphylococcus aureus.

${ }^{a}$ Includes all patients in contact with an MRSA carrier for whom placement in isolation was delayed and patients treated in a foreign healthcare institution during the 6 months before admission.

b This entry totals more than $100 \%$, because patients could have more than one characteristic.

' Antibiotics exclude perioperative prophylaxis.

of indwelling devices. ${ }^{2,15-21}$ Also, the high proportion of patients with MRSA infection at the time MRSA was first detected in them $(43.1 \%)$ was in accordance with earlier reports. ${ }^{22}$ Because of the low prevalence of nosocomial MRSA carriage (3\%-5\% of patients) and the virtual absence of community-acquired MRSA at this institution, the likelihood of a new and inadvertent colonization during follow-up was minimal. The study has some limitations, however. The retrospective study design precluded a strict schedule of followup screenings and randomized the administration of decolonization treatment. Also, we cannot exclude the possibility that study patients may have received MRSA-eradication regimens at another healthcare institution during follow-up.

Fifty-nine percent of the study patients cleared MRSA within a median interval of 226 days ( 7.4 months). Two previous studies found a similar time to clearance of 7-8.5 
TABLE 2. Determinants of Clearance of Methicillin-Resistance Staphylococcus aureus (MRSA) Colonization in 116 Patients With Newly Detected MRSA Carriage

\begin{tabular}{|c|c|c|c|c|}
\hline \multirow[b]{2}{*}{ Variable } & \multicolumn{2}{|c|}{ Clearance of MRSA } & \multirow[b]{2}{*}{$\mathrm{HR}(95 \% \mathrm{CI})$} & \multirow[b]{2}{*}{$P$} \\
\hline & $\begin{array}{c}\text { Yes } \\
(n=68)\end{array}$ & $\begin{array}{c}\text { No } \\
(n=48)\end{array}$ & & \\
\hline Age, $y$ & $53.6 \pm 21.1$ & $58.8 \pm 21.9$ & $1.00(0.99-1.01)$ & NS \\
\hline Female sex & $20(29.4)$ & $16(33.3)$ & $0.92(0.54-1.54)$ & NS \\
\hline \multicolumn{5}{|l|}{ No. of risk factors at first MRSA detection } \\
\hline Overall & $2.86 \pm 1.22$ & $3.69 \pm 1.52$ & $0.77(0.65-0.92)$ & .004 \\
\hline$>3$ risk factors & $25(36.8)$ & $27(56.3)$ & $0.62(0.38-1.01)$ & .06 \\
\hline$\geqslant 1$ modifiable risk factor & $60(88.2)$ & $48(100)$ & $0.20^{\mathrm{a}}(0.09-0.42)$ & $<.001$ \\
\hline Antibiotic therapy in past $30 \mathrm{~d}$ & $33(48.5)$ & $33(68.7)$ & $0.61(0.37-0.99)$ & .04 \\
\hline Skin lesion & $49(72.0)$ & $34(70.8)$ & $1.13(0.66-1.94)$ & NS \\
\hline Indwelling device & $27(39.7)$ & $26(54.2)$ & $0.70(0.42-1.18)$ & NS \\
\hline Surgery in past $30 \mathrm{~d}$ & $38(55.9)$ & $25(52.1)$ & $1.11(0.68-1.82)$ & NS \\
\hline Immunosuppressive therapy & $8(11.8)$ & $15(31.3)$ & $0.49^{\mathrm{a}}(0.23-1.02)$ & .05 \\
\hline Diabetes mellitus & $11(16.2)$ & $12(25.0)$ & $0.75(0.39-1.45)$ & NS \\
\hline Renal insufficiency & $10(14.7)$ & $11(22.9)$ & $0.66(0.33-1.30)$ & NS \\
\hline Hemodialysis & $1(1.5)$ & $6(12.5)$ & $0.08^{a}(0.01-0.66)$ & .01 \\
\hline Renal transplantation & $5(7.4)$ & $5(10.4)$ & $0.83(0.33-2.08)$ & NS \\
\hline Tumor & $12(17.7)$ & $11(22.9)$ & $0.86(0.46-1.61)$ & NS \\
\hline \multicolumn{5}{|l|}{ MRSA infection } \\
\hline At start & $35(51.4)$ & $15(31.3)$ & $1.53(0.95-2.46)$ & .08 \\
\hline During follow-up & $5(7.4)$ & $12(25.0)$ & $0.27(0.11-0.69)$ & .006 \\
\hline Decolonization treatment & $29(42.6)$ & $7(14.6)$ & $2.22^{a}(1.36-3.64)$ & .01 \\
\hline Intermittent screenings with negative results & $10(14.7)$ & $20(41.7)$ & $0.34(0.17-0.67)$ & .002 \\
\hline \multicolumn{5}{|l|}{ Risk factor at last MRSA screening } \\
\hline Overall no. & $1.06 \pm 1.17$ & $2.14 \pm 1.54$ & $0.73(0.59-0.90)$ & .003 \\
\hline Antibiotic therapy & $7(10.2)$ & $17(35.4)$ & $0.38(0.18-0.84)$ & .01 \\
\hline Immunosuppressive therapy & $8(11.8)$ & $14(29.2)$ & $0.45(0.22-0.95)$ & .03 \\
\hline Hemodialysis & $1(1.5)$ & $6(12.5)$ & $0.19(0.03-1.37)$ & .09 \\
\hline
\end{tabular}

NOTE. Data are no. (\%) of patients or mean value $\pm \mathrm{SD}$. CI, confidence interval; HR, hazard ratio; NS, nonsignificant $(P>.05)$.

${ }^{a}$ Adjusted HR estimates are presented for the following independent determinants: modifiable risk factors, receipt of hemodialysis, and receipt of immunosuppressive therapy at time of first MRSA detection and decolonization treatment. Crude estimates are presented for all other variables.

months. ${ }^{7,23}$ However, longer durations of 14 months ${ }^{8}$ and 40 months ${ }^{6}$ have been reported. These differences may be explained by the frequency of infection control screening performed in the different studies. For example, Vriens et al. ${ }^{8}$ screened patients at intervals of 6 months only. A mean of 1.6 screenings per patient per year were performed in the study by Sanford et al., ${ }^{6}$ whereas we performed a mean of 3.8 screenings per patient per year. A higher frequency of screenings certainly leads to a more accurate description of the duration of colonization. Estimates of the duration of colonization may also be influenced by the study population's size (49-197 patients were involved in the cited studies) and clinical characteristics. An indication for the latter might be the proportion of long-term carriers. In our study, approximately $20 \%$ of patients were long-term carriers (defined as carriage of MRSA for more than 1 year), and the longest observed carriage time was 3.3 years. Earlier studies reported a lower frequency of long-term carriage of approximately $10 \%{ }^{6,23,24}$ Lastly, different definitions used for MRSA clear- ance may also play a role. As in our study, Sanford et al. ${ }^{6}$ required 2 consecutive screenings with negative results; elsewhere, Vriens $\mathrm{et}^{\mathrm{a}} \mathrm{l}^{8}$ required 1 screening with a negative result, and no definition was given in the study by MacKinnon and Allen. ${ }^{23}$

Thirty of our 116 patients had at least 1 intermittent screening with negative results, and in $10 \%$ we observed 2 or more consecutive screenings with a negative result. Data on negative results of intermittent screenings are rare. Vriens et al. ${ }^{8}$ found $6.7 \%$ of their cohort to have negative results of intermittent screening, and in the study by Blok et al..$^{25}$ culturenegative intervals of 8-10 months were observed for 5 of 11 patients with long-term carriage. Low colonization density or an intracellular reservoir may be responsible for negative results of intermittent screenings. ${ }^{26,27}$ Interestingly, infection during follow-up was more frequent among patients with intermittent carriage, a finding that, to our knowledge, has not been reported up to now. Eventually, negative results of intermittent screenings may lead to a premature suspension 


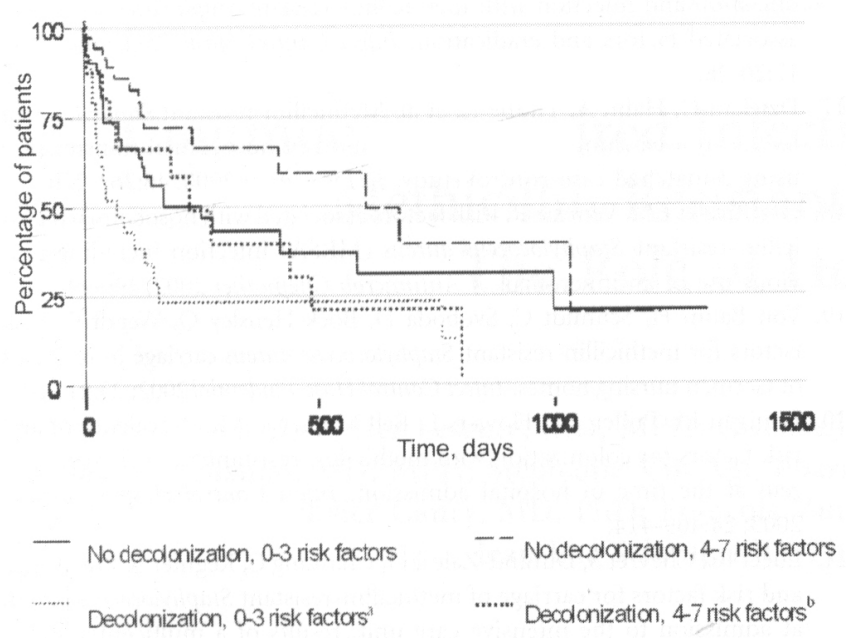

FIGURE. Kaplan-Meier curves for the time to methicillin-resistant Staphylococcus aureus (MRSA) clearance, stratified by decolonization treatment and the presence of risk factors at the time of MRSA detection. ${ }^{a}$ Log-rank test for decolonization treatment versus no decolonization treatment in patients with $0-3$ risk factors $(P=.02)$. ${ }^{b} \mathrm{Log}$-rank test for decolonization treatment versus no decolonization treatment in patients with 4-7 risk factors $(P=.03)$.

of infection control measures. In our study, however, the median interval to the first intermittent screening with a negative result was considerably shorter ( 31.5 days) than the observed median time to MRSA clearance (226 days).

Risk factors for MRSA colonization had a significant impact on the duration of MRSA colonization. The strength of this study is the detailed analysis of a large number of risk factors. The presence of at least 1 modifiable risk factor (antibiotic use, presence of a skin lesion, and use of an indwelling device), receipt of immunosuppressive therapy, and receipt of hemodialysis was independently associated with a longer duration of MRSA carriage. Earlier studies found skin lesions to be the most prominent factor associated with persistent carriage. $^{7,8,23,25,28}$ Beaujean et al. ${ }^{28}$ found an association between underlying disease and persistent carriage, albeit in a small study population. Cystic fibrosis was identified as a risk factor for long-term MRSA carriage in 2 studies ${ }^{8,24}$ but was only present in one of our patients. All these findings suggest that the presence of risk factors should be considered in the decision to suspend isolation precautions. Beaujean et al. ${ }^{28}$ demanded an absence of risk factors for more than 6 months and 3 screenings with negative results before ending isolation precautions. By comparison, Vriens et al. ${ }^{8}$ called for 12 months and at least 1 screening with a negative result. On the basis of our findings, with an extended analysis of risk factors for MRSA carriage, we propose that a patient should be considered to have cleared MRSA colonization if at least 2 consecutive screenings have negative results and if there are no other risk factors (modifiable and nonmodifiable), regardless of the time that has passed since MRSA colonization was detected. In the presence of long-term, nonmodifiable risk factors, a minimum follow-up duration (eg, 6 months) should be allowed to elapse, to decrease the risk of falsenegative results of intermittent screenings.

Receipt of decolonization treatment was significantly associated with the clearance of MRSA. However, it must be taken into consideration that, in this study, decolonization treatment was only administered when modifiable risk factors for MRSA colonization (antibiotic treatment, use of indwelling devices, and presence of skin lesions) were absent. Nevertheless, a high failure rate of decolonization treatment was observed (36.1\% of patients), and failure was associated with the presence of nonmodifiable risk factors for MRSA colonization. Vriens et al. ${ }^{8}$ reported a much higher success rate for decolonization treatment and concluded that such treatment should be administered to all patients, independent of the presence of risk factors. However, the large screening intervals used in their study and the lack of information on nonmodifiable risk factors precludes a comparison with our findings. We suggest that decolonization treatment should only be given when modifiable risk factors are largely absent and that the success of the treatment should be evaluated more carefully if long-term risk factors are present.

MRSA infection occurred in $14.7 \%$ of our patients during follow-up. This rate is comparable with rates reported elsewhere. ${ }^{1,29}$ Older age, intensive care unit stay, receipt of hemodialysis, presence of surgical wounds, presence of pressure ulcers, and use of intravascular catheters have been associated with an increased progression of MRSA colonization to invasive infection. ${ }^{1,29,30}$ In our study, a higher number of risk factors at the end of follow-up was associated with an increased risk of MRSA infection.

In conclusion, modifiable and nonmodifiable risk factors for MRSA acquisition are the most important determinants of the duration of MRSA colonization and affect the success rate of decolonization treatments. Moreover, the presence of risk factors may lead to intermittent screenings with negative results. Risk factors should therefore be evaluated carefully in all MRSA carriers and should be considered in infection control policies, such as the timing of decolonization treatment and the definition of MRSA clearance, which is the basis for the decision to suspend isolation measures.

\footnotetext{
Address reprint requests to Kathrin Mühlemann, $\mathrm{MD}, \mathrm{PhD}$, Institute for Infectious Diseases, University of Bern, Friedbühlstrasse 51, 3010 Bern, Switzerland (kathrin.muehlemann@ifik.unibe.ch).
}

\section{ACKNOWLEDGMENTS}

We thank Linda Beul, Susanne Burri, Rolf Kuhn, and Tanja Löhri for help with data collection and for stimulating discussions.

\section{REFERENCES}

1. Davis KA, Stewart JJ, Crouch HK, Florez CE, Hospenthal DR. Methicillin-resistant Staphylococcus aureus (MRSA) nares colonization at hospital admission and its effect on subsequent MRSA infection. Clin Infect Dis.2004; 39:776-782. 
2. Fishbain JT, Lee JC, Nguyen HD, et al. Nosocomial transmission of methicillin-resistant Staphylococcus aureus: a blinded study to establish baseline acquisition rates. Infect Control Hosp Epidemiol 2003; 24:415-421.

3. Muto CA, Jernigan JA, Ostrowsky BE, et al. SHEA guideline for preventing nosocomial transmission of multidrug-resistant strains of Staphylococcus aureus and Enterococcus. Infect Control Hosp Epidemiol 2003; 24:362-386.

4. Garner JS. Guideline for isolation precautions in hospitals: the Hospital Infection Control Practices Advisory Committee. Infect Control Hosp Epidemiol 1996; 17:53-80.

5. Herr CE, Heckrodt TH, Hofmann FA, Schnettler R, Eikmann TF. Additional costs for preventing the spread of methicillin-resistant Staphylococcus aureus and a strategy for reducing these costs on a surgical ward. Infect Control Hosp Epidemiol 2003; 24:673-678.

6. Sanford MD, Widmer AF, Bale MJ, Jones RN, Wenzel RP. Efficient detection and long-term persistence of the carriage of methicillin-resistant Staphylococcus aureus. Clin Infect Dis 1994; 19:1123-1128.

7. Scanvic A, Denic L, Gaillon S, Giry P, Andremont A, Lucet JC. Duration of colonization by methicillin-resistant Staphylococcus aureus after hospital discharge and risk factors for prolonged carriage. Clin Infect Dis 2001; 32:1393-1398.

8. Vriens MR, Blok HE, Gigengack-Baars AC, et al. Methicillin-resistant Staphylococcus aureus carriage among patients after hospital discharge. Infect Control Hosp Epidemiol 2005; 26:629-633.

9. Harbarth S, Dharan S, Liassine N, Herrault P, Auckenthaler R, Pittet D. Randomized, placebo-controlled, double-blind trial to evaluate the efficacy of mupirocin for eradicating carriage of methicillin-resistant Staphylococcus aureus. Antimicrob Agents Chemother 1999; 43:1412-1416.

10. Parras F, Guerrero MC, Bouza E, et al. Comparative study of mupirocin and oral co-trimoxazole plus topical fusidic acid in eradication of nasal carriage of methicillin-resistant Staphylococcus aureus. Antimicrob Agents Chemother 1995; 39:175-179.

11. Tomic V, Svetina Sorli P, Trinkaus D, Sorli J, Widmer AF, Trampuz A Comprehensive strategy to prevent nosocomial spread of methicillinresistant Staphylococcus aureus in a highly endemic setting. Arch Intern Med 2004; 164:2038-2043.

12. Loeb M, Main C, Walker-Dilks C, Eady A. Antimicrobial drugs for treating methicillin-resistant Staphylococcus aureus colonization. Cochrane Database Syst Rev 2003; (4):CD003340.

13. Blanc DS, Pittet D, Ruef C, et al. Epidemiology of methicillin-resistant Staphylococcus aureus: results of a nation-wide survey in Switzerland. Swiss Med Wkly 2002; 132:223-229.

14. Fux CA, Uehlinger D, Bodmer T, Droz S, Zellweger C, Muhlemann K. Dynamics of hemodialysis catheter colonization by coagulase-negative staphylococci. Infect Control Hosp Epidemiol 2005; 26:567-574.

15. Shimada M, Kamakura T, Itasaka H, Matsumata T, Hashizume M, Sugimachi K. The significance of methicillin-resistant Staphylococcus aureus infection in general surgery: a multivariate analysis of risk factors and preventive approaches. Surg Today 1993; 23:880-884.

16. Asensio A, Guerrero A, Quereda A, Lizan M, Martinez-Ferrer M. Col- onization and infection with methicillin-resistant Staphylococcus aureus: associated factors and eradication. Infect Control Hosp Epidemiol 1996; $17: 20-28$

17. Dziekan G, Hahn A, Thune K, et al. Methicillin-resistant Staphylococcus aureus in a teaching hospital: investigation of nosocomial transmission using a matched case-control study. J Hosp Infect 2000; 46:263-270.

18. Graffunder EM, Venezia R. Risk factors associated with nosocomial methicillin-resistant Staphylococcus aureus (MRSA) infection including previous use of antimicrobials. I Antimicrob Chemother 2002; 49:999-1005.

19. Von Baum H, Schmidt C, Svoboda D, Bock-Hensley O, Wendt C. Risk factors for methicillin-resistant Staphylococcus aureus carriage in residents of German nursing homes. Infect Control Hosp Epidemiol 2002; 23:511-515.

20. Jernigan JA, Pullen AL, Flowers L, Bell M, Jarvis WR. Prevalence of and risk factors for colonization with methicillin-resistant Staphylococcus aureus at the time of hospital admission. Infect Control Hosp Epidemiol 2003; 24:409-414.

21. Lucet JC, Chevret S, Durand-Zaleski I, Chastang C, Regnier B. Prevalence and risk factors for carriage of methicillin-resistant Staphylococcus aureus at admission to the intensive care unit: results of a multicenter study. Arch Intern Med 2003; 163:181-188.

22. Coello R, Jimenez J, Garcia $M$, et al. Prospective study of infection, colonization and carriage of methicillin-resistant Staphylococcus aureus in an outbreak affecting 990 patients. Eur I Clin Microbiol Infect Dis 1994; 13:74-81.

23. MacKinnon MM, Allen KD. Long-term MRSA carriage in hospital patients. J Hosp Infect 2000; 46:216-221.

24. Frenay HME, Vandenbroucke-Grauls CMJE, Molkenboer MJCH, Verhoef $\mathrm{J}$. Long-term carriage and transmission of methicillin-resistant Staphylococcus aureus after discharge from hospital. J Hosp Infect 1992; 22:207-215.

25. Blok HEM, Vriens MR, Weersink AJL, Troelstra A. Carriage of methicillin-resistant Staphylococcus aureus (MRSA) after discharge from hospital: follow-up for how long? a Dutch multi-centre study. J Hosp Infect 2001; 48:325-327.

26. Crowcroft NS, Ronveaux O, Monnet DL, Mertens R. Methicillin-resistant Staphylococcus aureus and antimicrobial use in Belgian hospitals. Infect Control Hosp Epidemiol 1999; 20:31-36.

27. Clement S, Vaudaux P, Francois P, et al. Evidence of an intracellular reservoir in the nasal mucosa of patients with recurrent Staphylococcus aureus rhinosinusitis. J Infect Dis 2005; 192:1023-1028.

28. Beaujean DJMA, Weersink AJL, Blok HEM, Frenay HME, Verhoef $J$. Determining risk factors for methicillin-resistant Staphylococcus aureus carriage after discharge from hospital. I Hosp Infect 1999; 42:213-218.

29. Coello R, Glynn IR, Gaspar C, Picazo JJ, Fereres J. Risk factors for developing clinical infection with methicillin-resistant Staphylococcus aureus (MRSA) amongst hospital patients initially only colonized with MRSA. J Hosp Infect 1997; 37:39-46.

30. Muder RR, Brennen C, Wagener MM, et al. Methicillin-resistant staphylococcal colonization and infection in a long-term care facility. Ann Intern Med 1991; 114:107-112. 